\title{
El Síndrome Nefrótico y el Diagnóstico Genético en Pediatría
}

\author{
MARTA AZÓCAR P. \\ Profesor Asistente, Facultad de Medicina Universidad de Chile. \\ Nefrólogo Infantil, Hospital Luis Calvo Mackenna. \\ Financiamiento Proyecto Fondecyt 11090045.
}

\begin{abstract}
Inheritance Genetics of Nephrotic Syndrome

Steroid-Resistant Nephrotic Syndrome (SRNS) is found in approximately $20 \%$ of patients with Nephrotic Syndrome (NS). Podocyte-gen mutations are diagnosed in a half of these children. Nephrin (NPHS1), podocin (NPHS2) and Wilms tumor suppressor gene (WT1) are the most frequently founded mutations. These patients usually progress to End Stage Renal Disease (ESRD). Objective: Current concepts in genetic diagnostic of NS in pediatrics are presented. A local experience is analyzed. In chilean pediatric patients with SRNS, a mutational analysis of the NPHS1 and NPHS2 gene was carried out by direct sequencing of the coding regions following polymerase chain reaction (PCR) amplification of genomic leukocyte DNA with flanking intronic primers. For WT1 (exon 8 and exon 9), PCR of these fragments were done. Thirty-three patients were included, 17 males, $11,1 \pm 6.8$ years. $54 \%$ of them developed ESRD, 12 patients were transplanted at the time of the analysis, 5 were under dialysis therapy, and 16 children correspond to ESRD Stage 3 and 4. Genetic analysis showed a gen mutation in 9 patients, NPHS1 in 3 and NPHS2 in 6 of them. All genetic NS patients were cyclosporine-resistant. Post transplant relapse of NS was lower in genetic patients $(p<0.05)$. Conclusion: SRNS in children should be always evaluated from a genetic approach in order to avoid long-term immunosuppression, and to anticipate a clinical evolution after kidney transplantation.
\end{abstract}

(Key words: Nephrotic syndrome, genetics, mutation, nephrin, NPHS1, podocyn, NPHS2).

Rev Chil Pediatr 2011; 82 (1): 12-20

\section{RESUMEN}

En pediatría, el $20 \%$ de los pacientes portadores de Síndrome Nefrótico Idiopático son corticoresistentes (SNCR). Aproximadamente la mitad de ellos corresponden a mutaciones de genes que codifican proteínas del podocito. Las mutaciones más frecuentes corresponden al gen de la nefrina (NPHS1), la podocina (NPHS2) y del gen supresor del tumos de Wilms (WT1). Estas formas hereditarias no responden a tratamientos inmunosupresores y pueden progresar a enfermedad renal terminal (ERT). Objetivo: Revisar el estado actual del diagnóstico genético en Síndrome Nefrótico en niños, y presentar esta experiencia nacional de esta patología.

Trabajo recibido el 12 enero de 2011, devuelto para corregir el 30 de enero de 2011, segunda versión 14 de febrero de 2011, aceptado para publicación 21 de febrero de 2011.

Correspondencia a:

Dra. Marta Azócar P.

E-mail: maazocar@med.uchile.cl 
Para el estudio de pacientes pediátricos chilenos, se realizó análisis de mutación del gen NPH2 por secuenciación directa de la regiones codificantes por PCR para la amplificación del DNA genómico leucocitario con partidores de acompañamiento intrónico. Para nefrina se procedió a extraer el DNA genómico, y se realizó la búsqueda de mutaciones de NPHS 1 por secuenciación directa de los 29 exones codificantes y las uniones intrónicas adyacentes, mientras que para el estudio de WT1 se practicó el análisis mutacional de los exones 8 y 9 , realizado por secuenciación directa del producto amplificado de WT1-PCR. Se han estudiado 33 pacientes provenientes de 29 familias, 17 varones, edad 11,1 + 6,8 años. Dieciocho pacientes (54\%) evolucionaron a ERT. Doce pacientes estaban trasplantados, 5 en diálisis, y 16 estaban en etapas 3-4 de enfermedad renal crónica. El estudio genético identificó mutaciones en 9 pacientes (27\%), 3 correspondieron a NPHS1, 6 a NPHS2. Ningún caso con mutación respondió a tratamiento de Ciclosporina A (CsA), y las recaídas posttrasplante fueron significativamente menores en el grupo con mutación (+). Conclusión: Las mutaciones en estos genes deben ser estudiadas en cada niño con SNCR con el fin de evitar tratamientos prolongados e inefectivos, y anticipar la evolución después del trasplante renal.

(Palabras clave: Síndrome Nefrótico, mutación, genética, NPHS1, nefrina, NPHS2, podocina).

Rev Chil Pediatr 2011; 82 (1): 12-20

\section{Antecedentes Generales y Demográficos}

El síndrome nefrótico $(\mathrm{SN})$ idiopático en niños se asocia frecuentemente a enfermedad de cambios mínimos y habitualmente responde favorablemente al tratamiento con corticoides. Esta nefropatía afecta aproximadamente 1-2 por 100000 niños menores de 16 años ${ }^{1,2,3}$. El SN se divide tradicionalmente en 2 categorías en base a la respuesta al tratamiento con esteroides: síndrome nefrótico córtico sensible (SNCS) y síndrome nefrótico córtico resistente $(\mathrm{SNCR})^{1,2,3}$. Los primeros reportes estimaron que el SNCR se observa en aproximadamente en el $10 \%{ }^{4}$ de los niños con síndrome nefrótico idiopático, mientras que los estudios más recientes reportan un $27 \%$ de los niños con SN eran córtico resistentes ${ }^{5}$. En Chile los datos acerca del SN son escasos. Norero y cols publicaron en 1996 que de 96 niños aproximadamente el $10 \%$ era resistente al tratamiento con esteroides. El International Study of Kidney Diseases in Children (ISKDC) reportó que en el $78,1 \%$ de los niños con SN se observa enfermedad de cambios mínimos en la biopsia renal, los que en su mayoría responden al tratamiento con esteroides, y que aproximadamente el 10\% corresponde a glomérulo esclerosis focal y segmentaria (GEFS), los que no responden a los esteroides ${ }^{4}$.

El registro epidemiológico chileno (CESNO $2002^{7}$ informó que la población menor de 18 años de edad era de 5 millones. Según esta cifra, para una incidencia anual de 5-10 pacientes con SNCR por 100000 menores de 18 años, se espera encontrar un número aproximado de 50 a 100 niños portadores de esta enfermedad.

Los pacientes que responden al tratamiento tienen buen pronóstico a largo plazo ${ }^{8}$, mientras los que no responden a esteroides progresan a enfermedad renal crónica y eventualmente a enfermedad renal terminal ${ }^{9,10}$.

El reporte de la Rama de Nefrología de la Sociedad Chilena de Pediatría publicó que para 179 trasplantes renales realizados entre los años 1989-2002, un 10\% de ellos correspondían a $\mathrm{GEFS}^{11}$, durante el mismo período en nuestro centro, el $17 \%$ de 100 trasplantes renales fueron debido a GEFS ${ }^{12}$.

\section{Tratamiento Médico}

El SN se caracteriza por una pérdida selectiva de la permeabilidad glomerular, con la consecuente proteinuria masiva, alteraciones del metabolismo proteico, lipídico, y algunos ejes del sistema endocrino ${ }^{1,11,13}$. Existen múltiples terapias publicadas para el manejo de esta enfermedad, sin embargo existe consenso respecto a que los corticoides son la primera línea de tratamiento ${ }^{14}$. El tratamiento de los SNCR plantea un desafío para los nefrólogos infantiles. Dado el mal pronóstico de esta forma de SN estos pacientes habitualmente son some- 
tidos a prolongados y agresivos tratamientos, con el objetivo de obtener la remisión de la enfermedad. Hasta hoy no se dispone de un marcador que logre predecir la respuesta a corticoides en niños con $\mathrm{SN}$, siendo el marcador pronóstico más confiable la respuesta al tratamiento corticoidal ${ }^{1,11}$. Los tratamientos que se han utilizado: corticoides, alquilantes (ciclofosfamida y clorambucil) por vía oral o vía endovenosa, micofenolato, ciclosporina o tacrolimus $^{1,15}$, buscan tener un efecto a nivel inmunológico, sin embargo, la mayoría de estos pacientes muestran progresión a enfermedad renal terminal, diálisis y trasplante renal. Estos tratamientos no están exentos de numerosos efectos adversos ${ }^{1,11,16}$. Los agentes alquilantes tienen efectos adversos como cistitis hemorragica, caida del pelo, toxicidad gonadal y enfermedades cancerígenas. Datos de 38 estudios que incluian a 1504 niños, quienes recibieron 1573 tratamientos de ciclofosfamida o clorambucil reportaron muerte en el $0,8 \%$, cistitis hemorragica en $2,2 \%$ e infecciones severas en el $1,5 \%$ de los pacientes tratados con ciclofosfamida, en tanto que los tratados con clorambucil presentaron $1,1 \%$ de muertes, $6,3 \%$ de infecciones y convulsiones en el 3,4\% de los pacientes ${ }^{1,11}$. Tanto un estudio americano ${ }^{17}$ como de un grupo francés ${ }^{18}$, y estudios más pequeños ${ }^{19}$, han reportado efectos adversos como hipertensión $28-29 \%$, hiperplasia gingival en $10-25 \%$, y disminución de la función renal con el uso de ciclosporina. Asociado al uso de tacrolimus se han descrito hiperglicemias transitorias, disminución de la función renal, y en menor frecuencia que con la ciclosporina, efectos cosméticos como hipertricosis e hiperplasia gingival ${ }^{20,21}$. Igualmente se han publicado reportes con grupos de pacientes tratados con micofenolato, correspondiendo a niños que no respondieron a inhibidores de la calcineurina, donde se observó como principal efecto colateral intolerancia digestiva $(21 \%)$, infecciones severas recurrentes $(1,9 \%)$, trombocitopenia y leucopenia moderadas $(1,9 \%)^{22,23}$. También se ha reportado respuesta favorable en algunos pacientes resistentes a las terapias previas con el uso de rituximab, comunicándose efectos adversos como reacciones mucocutáneas severas, reacciones fatales asociadas a la infusión, leucoencefalopatía progresiva multifocal, perforación intestinal y casos de enfermedad gastrointestinal severa inmunológica ${ }^{24}$.

\section{La Membrana Basal Glomerular}

Cada glomérulo está formado por la cápsula de Bowman y los capilares glomerulares, que están dentro de la cápsula. La cápsula de Bowman consta de dos capas de células epiteliales: la capa visceral y la capa parietal. La capa visceral está formada por los podocitos que emiten prolongaciones o pedicelos que se interdigitan y envuelven a los capilares glomerulares. Entre pedicelos vecinos y sus interdigitaciones quedan pequeñas ventanas o espacios recubiertos de lámina basal denominados ventanas de filtración. Entre los pedicelos y el endotelio de los capilares glomerulares se ubica la lámina basal, secretada por los podocitos y el endotelio capilar. La capa parietal de la cápsula de Bowman es un epitelio plano, que se continua posteriormente con el túbulo renal en el polo urinario. Entre ambas capas se encuentra el espacio urinario o de Bowman, que recibe el ultrafiltrado glomerular ${ }^{16,25}$. La lámina basal esta interpuesta entre los pedicelos de los podocitos y el endotelio capilar, y consta de tres capas: la lámina rara interna que está en contacto con el endotelio capilar, la lámina densa y la lámina rara externa, en contacto con los pedicelos. La lámina basal corresponde a matriz extracelular y está formada por fibras de colágeno (principalmente tipo IV), proteoglicanos polianiónicos y otras glicoproteinas. El conjunto formado por los pedicelos, las ventanas de filtración, la lámina basal y el endotelio fenestrado se conoce como barrera de filtración glomerular. Una serie de glomerulopatías causan alteraciones del tamaño de los poros o pérdida de componentes de la barrera de filtración glomerular, lo que trae como consecuencia un aumento su permeabilidad a las proteinas $^{6,25}$.

\section{Diafragma de Filtración}

Entre los procesos pedicelares que cubren la membrana basal glomerular se encuentran hendiduras de 25 a $60 \mathrm{~nm}$, cruzadas por una delga- 
da membrana llamada diafragma de hendidura o diafragma de filtración, estructura que en definitiva es la responsable de impedir el paso de moléculas como la albúmina ${ }^{29-31}$. El mayor componente del diafragma de filtración es la nefrina, codificada por el gen NPHS1, que se detalla más adelante. La nefrina podría interactuar con el centro proteico del diafragma de filtración, fundamentalmente con la P-cadherina. La P-cadherina tiene un dominio extracelular que forma parte del andamiaje del diafragma de filtración, mientras que su dominio intracelular está conectado con la -catenina y/o plakoglobina (-catenina). Estas proteinas interactúan con la cadherina intracitoplasmática que las une a la actina del citoesqueleto, y traducen señales intercelulares. Es a través de ellas que la nefrina regularía el tamaño de los poros y la permeabilidad selectiva del diafragma ${ }^{30-32}$. Otra proteina de alta importancia en la barrera de filtración glomerular es la podocina ${ }^{29,30}$, localizada en la fase citoplasmática del diafragma de filtración. Se encuentra junto con la CD2AP, proteína asociada al CD2, descrita originalmente en los linfocitos T. Esta proteína fija la nefrina al citoesqueleto de actina del diafragma, formando así el complejo nefrina-podocina-CD2AP del diafragma de filtración (figura 1).

\section{Mutaciones Genéticas Como Base de las Formas Clínicas del Síndrome Nefrótico}

En la última década se han descrito alteraciones genéticas ${ }^{19,20,23}$ como causa del SNCR. Dentro de las principales mutaciones asociadas a SNCR están el gen de la nefrina (NPHS1), el gen de la podocina (NPHS2) y el gen de WT1, uno de los principales mediadores de la diferenciación podocitaria. Estas dos primeras proteínas tienen un rol importante en la organización y funcionamiento del diafragma de filtración glomerular ${ }^{26}$.

En pacientes con síndrome nefrótico congénito y /o SNCR Ismaili ${ }^{15}$ reportó que el $81 \%$ de los pacientes eran portadores de una mutación (WT1, NPHS1, NPHS2).

El WT1 es un factor de transcripción, que se descubrió a través de clonamiento posicional en relación al tumor de Wilms ${ }^{27}$. Posteriormente se describieron mutaciones de WT1 en el síndrome de Frasier, síndrome de DenysDrash (DDS) y en la esclerosis mesangial difusa (DMS) con síndrome nefrótico aislado.

El DDS tiene herencia autosómica dominante y se caracteriza por tener un inicio temprano del síndrome nefrótico, pseudohermafroditismo masculino, disginesia gonadal y desarrollo del tumor de Wilms en un $90 \%$. La edad de inicio es en general el primer mes de vida. La histología renal muestra típicamente DMS y a la microscopía electrónica se observa una fusión de los pedicelos. El síndrome nefrótico es resistente al tratamiento con corticoides y hay un rápido deterioro de la función renal. La nefrectomía bilateral es recomendada por el alto riesgo de presentar un tumor de Wilms ${ }^{27}$. Las mutaciones se encuentran predominantemente en los exones 8 y 9 del gen WT1 y la mayoría son de mutaciones de novo no obser-

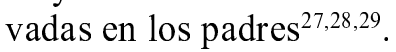

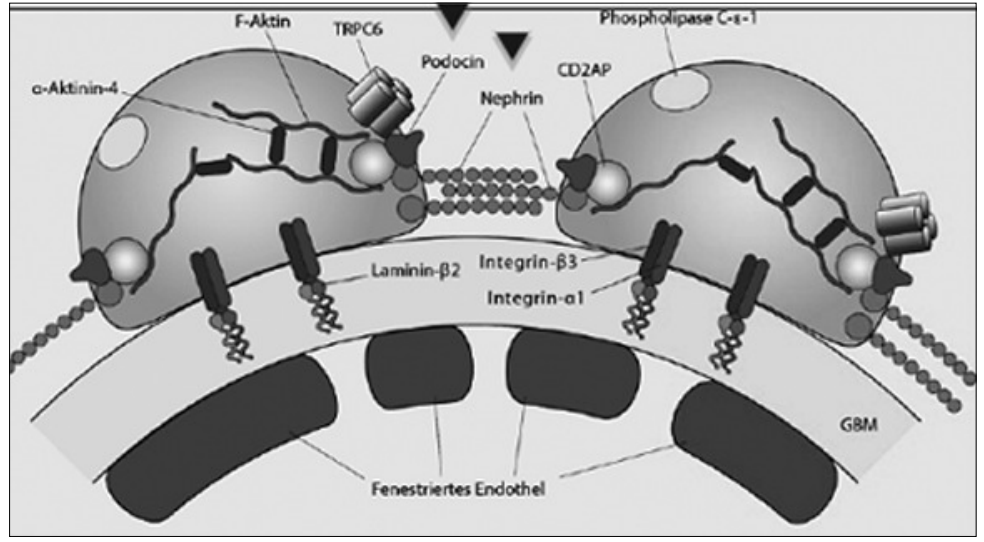

Figura 1 Barrera de filtración glomerular. Modificado de Weber S: Genetik des nephrotischen Syndroms. Der Nephrologe (2008); 5: 394-407 (29). 
Se ha observado que en algunos pacientes con mutaciones en el gen WT1 en los exones 8 y 9 no presentan todos las características del DDS sino que presentan una DMS aislada ${ }^{28}$. Es por esto que en los pacientes con DMS aislada debería realizarse el análisis del WT1 desde las fases tempranas del debut de síndrome nefrótico, debido al alto riesgo de desarrollar un tumor de Wilms en el caso que la mutación resulte positiva. Además se recomienda realizar un cariotipo en todas las niñas con DMS con el objetivo de detectar un pseudohermafroditismo masculino ${ }^{29}$.

El síndrome de Frasier (FS) se caracteriza por una glomerulopatía progresiva y un pseudohermafroditismo masculino, sin embargo se diferencia del DDS. La proteinuria se inicia más tardíamente durante la infancia y la velocidad de deterioro de la función renal es más lenta. $\mathrm{La}$ enfermedad renal terminal aparece en la segunda o tercera década de la vida. Al igual que en el DDS el síndrome nefrótico es córtico resistente. En el FS la histología renal típicamente muestra una GEFS y en una minoría se observa enfermedad de cambios mínimos. En pacientes de sexo femenino el tracto genitourinario está desarrollado en forma normal, mientras que en los pacientes 46XY se observa un sexo reverso completo con disginesia gonadal. Las mutaciones del gen WT1 en el FS se diferencian de las mutaciones del DDS: mientras que las mutaciones del DDS afectan la secuencia codificadora de los exones 8 y 9 , las mutaciones que se asocian al FS están localizadas en el intron 9. En forma similar a lo que ocurre en el DDS, las mutaciones que ocurren en FS son heterocigotas y frecuentemente se trata de mutaciones de novo, no observadas en los padres ${ }^{29}$.

El síndrome nefrótico congénito (SNC) tipo finés $(\mathrm{CNF})$ se caracteriza por tener una herencia autosómica recesiva y presentar proteinuria in útero. El gen responsable NPHS1 se logró localizar en 1994 en el cromosoma $19 \mathrm{q} 13^{16}$ y contiene 29 exones $^{29,30,31}$ y $26 \mathrm{~kb}$ de ADN genómico, y codifica para nefrina. La nefrina se expresa exclusivamente en los podocitos a nivel del diafragma de filtración glomerular después que ocurre la diferenciación. Pertenece a la superfamilia de las inmunoglobulinas, tiene un dominio transmembrana, un
$\mathrm{N}$-terminal intracelular corto y un C-terminal largo extracelular ${ }^{29,31}$. El C-Terminal extracelular une los espacios intercelulares entre los pedicelos, siendo un componente clave en el diafragma de filtración glomerular. La nefrina contribuye a la estructura porosa del diafragma de filtración glomerular, formando poros de aproximadamente $40 \mathrm{~nm}$. Estos poros son en parte responsables de la permeabilidad por tamaño del diafragma de filtración glomerular. Además de su rol como proteína estructural, nefrina también participa en las señales intracelulares, manteniendo la funcionalidad integral del podocito ${ }^{29,31}$ (figura 1).

Típicamente la enfermedad se manifiesta como un síndrome nefrótico severo que se presenta antes de los 3 meses de vida; la biopsia renal muestra glomérulos inmaduros, hipercelularidad mesangial, desaparición pedicelar y dilataciones pseudoquísticas del túbulo proximal. El síndrome nefrótico es córtico resisten$\mathrm{te}^{29,32}$. La nefrina es una proteína que tiene un dominio transmembrana con 8 porciones tipo Ig hacia el extracelular, una porción tipo fibronectina III y un C- terminal citosólico. Homodímeros y heterodímeros de la nefrina, junto con la proteína glomerular NEPH1 constituyen la base estructural del diafragma de filtración ${ }^{32}$. Además de su rol como proteína estructural, la nefrina también participa en las vía de señales intracelulares manteniendo la integridad funcional del podocito ${ }^{29,33}$. Las mutaciones en NPHS1 fueron por primera vez identificadas en la población finlandesa, por este motivo se denominó síndrome nefrótico "tipo fines"29,32. Dos mutaciones fueron encontradas con alta frecuencia en los niños finlandeses afectados, a las cuales se denominó: Fin mayor, (tiene alterada la proteína en su mayor parte) y Fin menor, (tiene alterada la proteína sólo en la parte del C- terminal). En los siguientes estudios las mutaciones del NPHS1 también fueron identificadas en pacientes no finlandeses, hasta el momento se han descrito más de 50 mutaciones diferentes ${ }^{29}$.

Estudios recientes sugieren que el CNF podría ser un trastorno genético heterogéneo: en pacientes afectados en los que no se encontraron las mutaciones del NPHS1, se encontró algunas mutaciones del NPHS2. Estos pacientes 
muestran características histológicas típicas del CNF. Sin embargo, estos resultados son preliminares y estudios adicionales se necesitarán con un mayor número de pacientes para confirmar el rol del NPH2 en el CNF ${ }^{31}$.

El gen de la podocina (NPHS2) se logró descubrir a través del análisis de ligamiento en 8 familias con SNCR con un patrón autosómico recesivo en el cromosoma 1q25-q31 y las mutaciones recesivas en NPHS2 fueron identificadas posteriormente ${ }^{34}$. El SN en estas familias se caracterizaba por ser córtico resistente, tener una edad de inicio entre los 3 y 5 años de edad y un bajo riesgo de recidiva de la proteinuria después del trasplante renal ${ }^{35}$. Las mutaciones de NPHS2 nunca han sido reportadas en pacientes con síndrome nefrótico córtico sensible. La histología renal típicamente muestra GEFS, sin embargo, algunos pacientes se presentan con lesiones de cambios mínimos ${ }^{34}$.

El gen NPHS2 codifica para podocina, proteína integral de membrana de $42 \mathrm{kD}$ que se expresa tanto en el glomérulo fetal como maduro. La podocina interactúa con nefrina y CD2AP, aparentemente uniendo a la nefrina al citoesqueleto podocitario. En pacientes que están afectados por una mutación recesiva en la NPHS2, la formación del diafragma de filtración glomerular esta alterado y hay desaparición pedicelar ${ }^{29}$.

Hasta el momento más de 30 mutaciones patogénicas han sido descritas en la NPHS2, la mayoría de ellas afectan el dominio del estoma, localizado en la parte del C-terminal de la proteína ${ }^{29,36}$. Las primeras mutaciones identificadas en NPHS2 fueron identificadas en niños con SNCR y progresión rápida a enfermedad renal terminal ${ }^{24}$, sin embargo, se hizo luego evidente, que defectos en la podocina pueden ser responsables de SNCR a diferentes edades, desde el nacimiento hasta la edad adulta $^{37,38}$, con evolución variable. Estudios recientes sugieren que mutaciones en NPHS2 están presentes en un $20-30 \%$ de niños con SNCR esporádicos ${ }^{36}$. Ruf et al ${ }^{36}$ reportaron que en 165 familias con SNCR, el 26\% mostró tener mutaciones homocigotas o heterocigotas compuestas en el gen NPH2 (podocina). En contraste no se encontraron mutaciones en 120 familias con síndrome nefrótico córtico sen- sible. Es destacable el hecho de que ninguno de los pacientes con mutaciones homocigotas o heterocigotas compuestos en el gen NPHS2 que fueron tratados con ciclosporina o ciclofosfamida presentó remisión completa del síndrome nefrótico ${ }^{36}$.

La variación geográfica tiene importancia en las mutaciones de NPHS2. Fuchshuber y $\operatorname{cols}^{34}$, encontraron mutaciones de la NPH2 en un $46 \%$ de las familias estudiadas, mientras que Maruyama et $\mathrm{al}^{38}$, reportaron que las mutaciones en el gen de NPHS2 son poco comunes en pacientes pediátricos japoneses con SNCR.

La mayoría de los estudios publicados se refieren a población europea y reportan que la mutación más frecuente en pacientes alemanes y franceses es la $\mathrm{R} 138 \mathrm{Q}^{39}$, mientras que la mutación P20L era más frecuentemente observada en pacientes de Italia ${ }^{40,41}$ y Turquía ${ }^{15}$. La edad del inicio de la proteinuria varía en los diferentes reportes ${ }^{13,34,40,41}$. Weber ${ }^{39}$ reportó que R138Q podría estar asociada a un inicio precoz y por otro lado V180M, R229Q y R238S podrían estar asociadas con un inicio tardío del síndrome nefrótico, en tanto que Berdeli et $\mathrm{al}^{42}$ reportaron que P20L y R168H se encuentran asociadas a inicio precoz de la enfermedad.

Se ha señalado que pacientes con SN esporádico que tienen mutaciones en NPHS2 progresan a enfermedad renal terminal después de los 73 meses desde el inicio de la proteinuria (rango 6-155 meses), mientras que los casos de SN familiares alcanzan la enfermedad renal crónica después de un seguimiento de 76 meses (rango 18-162 meses) $13,34,40,41$.

De esta forma, las mutaciones de podocina parecen ser responsables de algunos casos de SNCR tanto familiares como esporádicos. La heterogeneicidad étnica de esta enfermedad hace necesaria la identificación de las mutaciones de NPHS2 en pacientes de diferentes países, en Chile por ahora existen sólo limitados estudios publicados en la literatura ${ }^{43,44}$.

\section{Sindrome Nefrótico y Genética, la Experiencia Nacional}

Con el fin de revisar la experiencia chilena en relación al síndrome nefrótico y mutaciones genéticas en los últimos 15 años, se realizó 
una búsqueda en las bases de datos de Scielo y Pubmed, utilizando las palabras: Pediatrics, Children, Nephrotic syndrome, Nephrotics, Corticoresistant Nephrotic syndrome and Genetics, Nephrotic syndrome and Mutations, Nephrin, Podocin and WT1. Encontramos 7 artículos publicados después del año 1990 en la Revista Chilena de Pediatría, 3 de estos ${ }^{43,44,45}$ se relacionan con genética, sólo uno de ellos es en población pediátrica ${ }^{44}$ (caso clínico), y uno en adultos jóvenes ${ }^{43}$ relacionado con mutaciones en SN. No se encontró en la base de datos PubMed ningún artículo de Chile o de Latinoamérica. Podemos concluir que hasta el momento no existen estudios en nuestro país orientados al estudio de las bases genéticas del CRSN.

El estudio Fondecyt 11090045 "Caracterización genética de una población chilena de niños con síndrome nefrótico: del diagnóstico histológico al diagnóstico molecular" nos ha permitido estudiar la presencia de mutaciones para nefrina, podocina y WT1. El análisis de las mutaciones para los genes NPHS1, NPHS2 y para WT1 se realizó por reacción de polimerasa en cadena (PCR) con secuenciación directa de las regiones codificadoras, en labor conjunta entre el laboratorio de biología molecular del Hospital Luis Calvo Mackenna y el Laboratorio de Nefrología Infantil de la Universidad de Heidelberg, Alemania.

Los estudios realizados para Podocina (NPHS2) consideraron el análisis de mutación del gen NPH2 por secuenciación directa de la regiones codificantes por PCR para la amplificación del DNA genómico leucocitario con partidores de acompañamiento intrónico. $\mathrm{La}$ secuenciación del DNA fue realizada usando el método fluorométrico (Big-Dye Terminator Sequencing Kit; ABI 3700 DNA sequencer, Applied Biosystems, Foster City, CA, USA) de acuerdo a protocolos de fabricación, y las secuencias fueron evaluadas con el Programa SeqPilot (JSI Medical Systems GmbH Kip penheim, Germany). En el estudio de la Nefrina el DNA genómico fue extraído de sangre periférica por métodos estándar, y se realizó la búsqueda de mutaciones de NPHS1 por secuenciación directa de los 29 exones codificantes y las uniones intrónicas adyacentes, mientras que para el estudio de WT1 se practi- có el análisis mutacional de 8 y 9 exones, realizado por secuenciación directa del producto amplificado de WT1-PCR, como fue descrito por Jeanpierre y $\operatorname{cols}^{28}$.

Hasta el momento se han estudiado 33 pacientes chilenos con SNCR, 17 varones, edades 11,04 $\pm 6,77$ años; el diagnóstico histológico correspondió a glomerulo esclerosis focal y segmentaria en 19 casos, enfermedad de cambios mínimos 9, esclerosis global focal 2, otros 3. La edad al diagnóstico fue de 2.7 años $(0-9,5)$. Al momento del estudio 12 pacientes habían sido trasplantados, 5 permanecían en diálisis, y 16 estaban en etapas 3-4 de enfermedad renal crónica (IRC). El estudio genético identificó mutaciones en 9 pacientes $(27 \%)$, 3 correspondieron a NPHS1, 6 a NPHS2. El análisis genético por edad de debut del SNCR ( $<3 v s>3$ meses) identificó $3 / 5$ y 6/28 pacientes portadores de mutación respectivamente. $\mathrm{Al}$ analizar la respuesta a inmunosupresores, ninguno de los casos con mutación positiva respondió a tratamiento de Ciclosporina A (CsA), en tanto que en los pacientes con estudio negativo para las mutaciones se observó una respuesta favorable a CsA en el $70 \%$ de ellos (17/22).

Las mutaciones encontradas para nefrina fueron: C567X homocigoto, inserción de nucleótido 419 GCAG en exón 9 e inserción de nucleótido $1095 \mathrm{G}$ en exón 24. Las mutaciones para podocina fueron P341S homocigoto/ R229Q heterocigoto en un paciente, A284V heterocigoto/R229Q heterocigoto en 5 pacientes. Para WT1 resultaron todos los estudios negativos.

En el grupo de pacientes con mutación, siete llegaron a enfermedad renal terminal (ERT) a la edad de $12,5 \pm 6,84$ años, vs 10/24 en el grupo sin mutación, que llegaron a ERT, cinco a los 6,9 $\pm 3,5$ años (p: n. s.).

En los pacientes trasplantados renales por SNCR, si los separamos en un grupo con mutación $v s$ otro sin mutación, 1/6 vs 5/7 respectivamente presentaron recaída del síndrome nefrótico después del trasplante.

El porcentaje de mutaciones en SNCR en casos familiares de NPHS2 fue de $60 \%$ vs un $10 \%$ en los casos esporádicos. Ninguno de estos pacientes respondió a Ciclosporina (CsA). 


\section{Conclusión}

Por primera vez en nuestro país se estudia a niños portadores de SNCR desde el punto de vista genético. El porcentaje de mutación en este estudio fue de un $27 \%$, cifra similar a series extranjeras. La mayoría de los casos correspondieron a mutación del gen NPHS2.

Las mutaciones en estos genes deben ser estudiadas en cada niño con SNCR con el fin de evitar una terapia innecesaria. El SNCR es una enfermedad llena de interrogantes que ilustra la relevante importancia clínica que el estudio genético puede tener en pacientes y familias afectadas por esta enfermedad. En los niños con una causa genética de SNCR se puede evitar el uso de tratamiento inmunosupresor ${ }^{1,35}$. El análisis de las mutaciones en los padres nos puede proporcionar las bases de un consejo genético respecto a la planificación familiar, pero también en el caso de ser donante relacionado de órganos ${ }^{29,35}$. Además el riesgo de recurrencia del síndrome nefrótico en el riñón trasplantado es mucho menor en pacientes que tienen alteraciones estructurales del podocito versus las formas inmunológicas del $\mathrm{SNCR}^{1,29,35}$. La implentación de laboratorios tanto de genética como de biología molecular en nuestro país son necesarios de implementar con las técnicas de reacción de polimerasa en cadena y secuenciación de las proteinas ya conocidas, y debemos apuntar a estudiar a nuestra población y asi determinar si para nuestra diversidad étnica son las técnicas suficientes de estudio o se deben buscar nuevos genes que esten presentes a nivel local.

\section{Referencias}

1.- Hodson E: The management of idiopathic Nephrotic Syndrome in Children. Paediatric Drugs 2003; 5: 335-49.

2.- Chernin G, Heeringa $S$, Gbadegesin, et al: Low prevalence of NPHS2 mutation SN in African American children with steroid-resistant nephrotic syndrome. Pediatr Nephrol 2008; 23: 1455-60.

3.- Schwaderer P, Knuppel T, Honrad M, et al: Clinical course and NPHS2 analysis in patients with late steroid-resistent nephrotic syndrome. Pediatr Nephrol 2008; 23: 251-6

4.- International Study of Kidney Disease in Children: The primary nephrotic syndrome in children: Clinical significance of histopathologic variant of minimal change and of diffuse mesangial hypercellularity: A report of the International Study of kidney disease in children. Kidney Int 1981; 20: 765-71.

5.- Kim J, Belew C, Silverstein D, Aviles D, Boineau F, Vehaskari $V$ : High incidence of initial and late steroid resistance in childhood nephrotic syndrome. Kidney Int 2005; 68: 1275-81.

6.- Norero C, Delucchi A, Lagos E, Rosati P. Cuadro inicial del síndrome nefrótico primario del niño: evaluación a 18 meses de dos esquemas de tratamiento con prednisona. Rev Med Chile 1996; 124: 567-72.

7.- $\quad$ http://www.ine.cl/cd2002/sintesiscesnal.pdf

8.- Cattran DC, Rao P: Long-term outcome in children and adults with classic focal segmental glomerulosclerosis. Am J Kidney Dis 1998; 32: 72-9.

9.- Schaerer K, Minges U: Long term prognosis of the nephrotic syndrome in childhood. Clin Nephrol 1973; 1 : 182-7.

10.- McBryde KD, Kershaw DB, Smoyer WE: Pediatric steroid-resistant nephrotic syndrome. Curr Probl Pediatr Adolesc Health Care 2001; 31: 280-307.

11.- Rosati P, Pinto V, Delucchi A, et al: Pediatric renal trasnplantation: 13 years of Experience from the Chilean cooperative multicenter group. Trasnplant Proc 2005; 37: 1569-73.

12.- Delucchi A, Ferrario M, Varela $M$, et al: Pediatric renal transplantation: a single center experience over 14 years. Pediatr Transplant 2006; 10: 193-7.

13.- Hodson E, Craig J: Therapies for steroid-resistant nephrotic syndrome. Pediatr Nephrol (2008); 23: 13914.

14.- Brodehl J, Krohn HP, Ehrich JH: The treatment of minimal change nephrotic syndrome (lipoid nephrosis) Cooperative studies of the Arbeitsgemeinschaft fuer Paediatrische Nephrologie (APN), Klin Padiatr 1982; 194: 162-5.

15.- Ismaili K, Pawtowski A, Boyer O, Wissing K, Janssen F, Hall $M$ : Genetic forms of nephrotic syndrome: a singlecenter experience in Brussels. Pediatr Nephrol 2009; 24: 287-94.

16.- Pollak M: Inherited Podocytopathies: GEFS and nephrotic syndrome from a genetic viewpoint. J Am Soc Nephrol 2002; 13: 16-23.

17.- Singh A, Tejani C, Tejani A: One-center experience with cyclosporine in refractory nephrotic syndrome in children. Ped Neph 1999; 13: 26-32.

18.- Niaudet $P$ : Treatment of childhood corticosteroid-resistant idiopathic nephrosis with combination of cyclos- 
porine and prednisone the French Society of Pediatric Nephrology. J Pediatr 1994; 125: 981-6.

19.- McBryde KD, Kershaw DB, Somer WE: Pediatric corticosteroid-resistant nephrotic syndrome. Curr Probl Pediatric 2001; 31: 275-307.

20.- Roberti I, Vyas S: Long term outcome of children with steroid resistant nephrotic syndrom treated with tacrolimus. Ped Neph 2010; 25: 1117-24.

21.- Choudhry S, Bagga A, Hari P, Sharma S, Kalaivani M, Dinda $A$ : Efficacy and safety of tacrolimus versus cycloporine in children with steroid- resistant nephrotic syndrome: radomized controlled trail. Am J Kidney Dis 2009; 53:760-9.

22.- Moudgil, A Bagga A, Jordan S: Mycophenolate mofetil therapy in frequently relapsing steroid-dependent and steroid-resistant nephrotic syndrome of childhood: current status and future direction (2005); Ped Neph 20: 1376-81.

23.- de Mello VR, Rodrigues MT, Mastrocinque TH, et al: Mycophenolate mofetil in children with steroid/cyclophosphamide-resistant nephrotic syndrome. Ped Neph 2010; 25: 453-60.

24.- Ardelean DS, Gosnka T, Wires $S$, et al: Severe ulcerative colitis after rituximab therapy. Pediatrics 2010; 126: 243-6.

25.- Huber TB, Hartleben B, Kim J et al: Nephrin and $\mathrm{CD} 2 \mathrm{AP}$ associate with phospoinositde 3-OH kinase and stimulate AKT-dependt signalling. Mol Cell Biol 2003; 23: 4917-28.

26.- Niaudet $P$ : WT1 and glomerular diseases. Pediatr Nephrol 2006; 21: 1653-60.

27.- Jeanpierre C, Denamur E, Henry I, et al: Identification of constitucional WT1 mutation, in patients with Isolated Diffuse Mesangial Sclerosis, and analysis of Genotype/Phenotype Correlations by use of a computerized mutation Database. Am J Hum Genet 1998; 62: 824-33.

28.- Weber S: Genetik des nephrotischen Syndroms. Der Nephrologe 2008; 5: 394-407.

29.- Kestila M, Mannikko M,Holmberg C et al: Congenital nephrotic syndrome of the Finnish type maps to the long arm of chromosome 19. Am J Hum Genet 1994; 54: 757-64.

30.- Philippe A, Nevo F, Esquivel E, et al: Nephrin mutations can cause childhood-onset Steroid-Resistant Nephrotic syndrome. J Am Soc Nephrol 2008; 19: 1871-8.

31.- Koziell A, Grech V, Hussain S et al: Genotype/phenotype correlation of NPHS1 mutation in nephrotic syndro- me advocate a functional inter-relationship in glomerular filtration. Hum Mol Genet 2002; 11: 379-88.

32.- Coulthard MG: Manegement of Finnish congenital nephritic syndrome by unilateral nephrectomy. Pediatr Nephrol 1989; 3: 451-53.

33.- Fuchshuber A, Jean G, Gribouval O, et al: Mapping a gene (SRN1) to chromosome 1q25-q31 in idiopathic nephritic syndrome confirms a distinct entity of autosomal recessive nephrosis. Hum Molec Genet 1995; 4: 2155-8.

34.- Weber S, Tonshoff B: Recurrence of focal-segmental glomerulosclerosis in children after renal transplantation: clinical and genetic aspects. Transplantation 2005; 80: S128-S133.

35.- Ruf RG, Lichtenberger A, Karle SM, et al: Patients with mutation in NPHS2 (podocin) do not respond to standard steroid treatment of nephrotic syndrome. J Am Soc Nephrol 2004; 15: 722-32.

36.- Karle SM, Uetz B, Ronner V, Glaeser L, Hildebrandt F, Fuchshuber: A novel mutation in NPHS2 detected in both familial and sporadic steroid-resistant nephrotic syndrome. J Am Soc Nephrol 2002; 13: 388-93.

37.- Maruyama K, Iijima $K$, Ikeda $M$, et al: NPH2 mutation in sporadic steroid-resistant nephrotic syndrome in Japanise children. Pediatr Nephrol 2003; 18: 412-16.

38.- Weber S, Gribouval O, Esquivel, et al: NPH2 mutation analysis shows genetic heterogeneity of steroid-resistant nephrotic syndrome and low post-transplant recurrence. Kidney Int 2004; 66: 571-9.

39.- Caridi G, Bertelli R, Di Duca M, et al: Broadening the spectrum of diseases related to podocin mutation. J Am Soc Nephrol 2003; 14: 278-86

40.- Caridi G, Perfumo F, Ghiggeri GM: NPHS2 (podocin) mutation in nephrotic syndrome. Clinical spectrum and fine mechanisms. Pediatr Res 2005; 57: 54-61.

41.- Berdeli A, Mir S, Yavascan O, et al: NNPHS2 (podocin) mutation in Turkish children with idiopathic nephrotic syndrome. Pediatr Nephrol 2007; 22: 2031-40.

42.- Ardiles L, Carrasco A, Carpio J, Mezzano S: Late onset of familial nephrotic syndrome associated with a compound heterozygous mutation of the podocin-encoding gene. Nephrology 2005; 10: 553-6.

43.- Pedraza GN, Ceballos OML, Cano Sch: Síndrome nefrótico cortico-resistente secundario a mutación genética, a propósito de 2 casos clínicos. Rev Chil Ped 2008; 79: 398-403.

44.- Norero $C$, et al: Síndrome nefrótico en el primer año de vida. Rev Chil Ped 1996; 67: 112-5. 